\title{
Transient Hypothyroxinemia
}

National Cancer Institute

\section{Source}

National Cancer Institute. Transient Hypothyroxinemia. NCI Thesaurus. Code C131031.

Reduced concentration of thyroid hormone(s), usually associated with a non-thyroidal illness, that resolves spontaneously. 